\title{
Approximate Confidence Interval for the Mean of Poisson Distribution
}

\author{
Manad Khamkong \\ Department of Statistics, Faculty of Science, Chiang Mai University, Chiang Mai, Thailand \\ Email:manad.k@cmu.ac.th
}

Received February 19, 2012; revised March 20, 2012; accepted April 5, 2012

\begin{abstract}
A Poisson distribution is well used as a standard model for analyzing count data. Most of the usual constructing confidence intervals are based on an asymptotic approximation to the distribution of the sample mean by using the Wald interval. That is, the Wald interval has poor performance in terms of coverage probabilities and average widths interval for small means and small to moderate sample sizes. In this paper, an approximate confidence interval for a Poisson mean is proposed and is based on an empirically determined the tail probabilities. Simulation results show that the proposed interval outperforms the others when small means and small to moderate sample sizes.
\end{abstract}

Keywords: Confidence Interval; Coverage Probability; Poisson Distribution; Expected Width; Wald Interval

\section{Introduction}

In many applications, the variable of interest is given in the form of an event count or a non-negative integer value which refers to the number of a occurrences of particular phenomenon over a fixed set of time, distance, area or space. Some examples of such data are number of road accident victims per week, number of cases with a specific disease in epidemiology, etc. Poisson distribution is a standard and good model for analyzing count data and it seems to be the most common and frequently used as well.

It is very interesting to construct a confidence interval for a Poisson mean. Suppose $\mathrm{X}_{1}, \mathrm{X}_{2}, \cdots, \mathrm{X}_{\mathrm{n}}$ is a random sample of size $\mathrm{n}$ from a Poisson $(\lambda>0)$ distribution. A problem in finding an exact $1-\alpha$ two-sided confidence interval $[\mathrm{L}(\mathrm{X}), \mathrm{U}(\mathrm{X})]$ for mean $(\lambda)$ of Poissonity is given by

$$
\begin{aligned}
& \mathrm{L}(\mathrm{X})=\mathrm{P}\left(\mathrm{X} \geq \mathrm{x} \mid \lambda=\lambda_{\mathrm{L}}\right)=\frac{\alpha}{2} \\
& \mathrm{U}(\mathrm{X})=\mathrm{P}\left(\mathrm{X} \leq \mathrm{x} \mid \lambda=\lambda_{\mathrm{U}}\right)=\frac{\alpha}{2},
\end{aligned}
$$

where $\lambda_{\mathrm{L}}$ and $\lambda_{\mathrm{U}}$ are, respectively, the lower and upper endpoints of the confidence interval.

Let $\hat{\lambda}=\overline{\mathrm{X}}=\mathrm{n}^{-1} \sum_{\mathrm{i}=1}^{\mathrm{n}} \mathrm{X}_{\mathrm{i}}$ is the maximum likelihood estimator of $\lambda$. As $n$ large by central limit theorem, the Wald interval for the mean is given by

$$
\overline{\mathrm{X}} \pm \mathrm{z}_{\alpha / 2} \sqrt{\frac{\overline{\mathrm{X}}}{\mathrm{n}}},
$$

where $\mathrm{z}_{\alpha / 2}$ is the $(1-\alpha / 2) 100^{\text {th }}$ percentile of the standard normal distribution. The Wald interval with continuity correction interval (WCC) uses a normal distribution to approximate a Poisson distribution is defined as

$$
\overline{\mathrm{X}} \pm \mathrm{z}_{\alpha / 2} \sqrt{\frac{\overline{\mathrm{X}}+0.5}{\mathrm{n}}},
$$

Several methods have been proposed to construct a confidence interval for a Poisson mean such as Cai [1], Byrne and Kabaila [2], Guan [3], Krishnamoorthy and Peng [4], Stamey and Hamillton [5], Swifi [6] and others. Guan [3] has suggested that the score interval (SC) is the uppermost approximation on interval estimation of a Poisson mean for moderate $\lambda$ is given by

$$
\overline{\mathrm{X}}+\frac{\mathrm{z}_{\alpha / 2}^{2}}{2 \mathrm{n}} \pm \mathrm{z}_{\alpha / 2} \sqrt{\frac{\overline{\mathrm{X}}+\frac{\mathrm{z}_{\alpha / 2}^{2}}{4 \mathrm{n}}}{\mathrm{n}}}
$$

and he has also proposed the moved score confidence interval (MSC) as follows,

$$
\overline{\mathrm{X}}+\frac{0.46 \mathrm{z}_{\alpha / 2}^{2}}{\mathrm{n}} \pm \mathrm{z}_{\alpha / 2} \sqrt{\frac{\overline{\mathrm{X}}+\frac{\mathrm{z}_{\alpha / 2}^{2}}{4 \mathrm{n}}}{\mathrm{n}}}
$$

Barker [7] has recommended the exact confidence interval outperform but not explicit closed form and was computed difficult. In particular, the Wald interval with continuity correction interval (WCC) achieves coverage probabilities quite faster than the Wald interval. However, The WCC is known to perform poorly for small to mod- 
erate sample sizes.

This paper interested in estimating the tail probabilities of the Wald interval that view be propose in the next section. The third section, the empirical results of the simulation studies are illustrated by the examples. Some concluding remarks appear in the last section.

\section{Proposed Confidence Interval}

The basic idea improvement on the Wald interval is being that the confidence interval should add the tail probbilities for small sample size adjusted by $\mathrm{c}=\frac{\mathrm{z}_{\alpha / 2}^{2}}{2 \mathrm{n}}$. Let $\mathrm{X}_{1}, \mathrm{X}_{2}, \cdots, \mathrm{X}_{\mathrm{n}}$ be independent and identically distributed random variables of size $\mathrm{n}$ selected from a Poisson distribution with mean $\lambda$ and $\mathrm{c}=\frac{\mathrm{z}_{\alpha / 2}^{2}}{2 \mathrm{n}}$. Then

1) $\mathrm{E}[\overline{\mathrm{X}}+\mathrm{c}]=\lambda+\mathrm{c}$ and $\mathrm{V}[\overline{\mathrm{X}}+\mathrm{c}]=\mathrm{n}^{-1} \lambda$

2) $\frac{\sqrt{\mathrm{n}}(\overline{\mathrm{X}}+\mathrm{c}-\lambda)}{\sqrt{\lambda}} \stackrel{\mathrm{D}}{\longrightarrow} \mathrm{N}(0,1)$ as $n \rightarrow \infty$

According to Equation (7), it is appropriated to proposed an approximate confidence interval for a Poisson mean, called adding the tail probability of the Wald interval (AWC) as follows,

$$
\overline{\mathrm{X}}+\frac{\mathrm{z}_{\alpha / 2}^{2}}{2 \mathrm{n}} \pm \mathrm{z}_{\alpha / 2} \sqrt{\frac{\overline{\mathrm{X}}}{\mathrm{n}}}
$$

For any nominal $(1-\alpha) 100 \%$ confidence interval for mean $(\lambda)$, the coverage probability at a fixed value of $\lambda$ is given by

$$
\mathrm{CP}(\lambda)=\sum_{\mathrm{i}=0}^{\infty} I_{\{\mathrm{L}(\mathrm{i}) \leq \lambda \leq \mathrm{U}(\mathrm{i})\}} \frac{\mathrm{e}^{-\lambda} \lambda^{\mathrm{i}}}{\mathrm{i} !}
$$

where $I_{\{\}}$is the indicator function of the bracketed event. Similarly the expected width of any confidence interval is

$$
\operatorname{EW}(\lambda)=\sum_{\mathrm{i}=0}^{\infty}[\mathrm{U}(\mathrm{i})-\mathrm{L}(\mathrm{i})] \frac{\mathrm{e}^{-\lambda} \lambda^{\mathrm{i}}}{\mathrm{i} !}
$$

\section{Empirical Results}

This section presents some selected empirical results for comparing the performance of the aforementioned confidence intervals for mean of Poisson distribution. The proposed confidence interval, AWC, will be compared with the other 3 intervals namely score interval (SC), the moved score confidence interval (MSC) and the Wald interval with continuity correction interval (WCC). The estimated coverage probabilities and the average widths of these intervals are evaluated by a Monte Carlo simulation using 50,000 replications for small to moderate sample sizes, $\mathrm{n}=15,25,50,100$ and the confidence interval level to be considered is $95 \%(1-\alpha=0.95)$, provided by the statistical package $\mathrm{R}$ [8]. For each sample is drawn from a Poisson distribution with mean parameter $\lambda=1,1.5,3,5,10$.

\subsection{Simulation Results}

The simulation results are reported in Table 1. All confidence intervals can control the coverage probabilities to be close enough to the 0.95 level except WCC can achieve coverage greater than nominal level for most values of $\lambda$. While an approximated confidence interval having some of coverage probabilities less than the nominal level when small means, $\lambda=1,1.5$ and small to moderate sample sizes, $\mathrm{n}=15,25,50$. The proposed interval (AWC) outperforms the others in terms of the maintain coverage probabilities and the average widths shorter than those of the other confidence intervals.

\subsection{Examples}

An example 1: numbers of sparrow nests found in one hectare area, $n=40$ areas of Zar (quoted in Gürtler and Henze [9]).

$$
\begin{aligned}
& \text { No. of nests: } \begin{array}{lllll}
0 & 1 & 2 & 3 & 4
\end{array} \\
& \text { Frequency: } \begin{array}{llllll}
9 & 22 & 6 & 2 & 1
\end{array}
\end{aligned}
$$

The sample mean and variance are 1.1 and 0.8103 , respectively. Estimated tail probability is 0.0480 . That is, 95\% AWC confidence interval of the average sparrow nests found in one hectare area is between 0.823 and 1.473 and the average width is 0.65 .

An example 2: the annual number of serious earthquakes over a period of 75 years (1903-1977, quoted in Blaesild and Granfeldt [10]). An earthquake is considered serious if its magnitude is at least 7.5 on the Richter scale or if more than 100 people were killed.

$$
\begin{array}{llcccc}
\text { No. of serious earthquakes: } 0 & 1 & 2 & 3 & 4 \\
\text { Frequency: } & 31 & 28 & 14 & 1 & 1
\end{array}
$$

The sample mean and variance are 0.84 and 0.7578 , respectively. Therefore, $95 \%$ AWC confidence interval of the average number of serious earthquakes per year is between 0.6582 and $1.0730(\hat{\mathrm{c}}=0.0256$ and $\operatorname{EW}(\hat{\lambda})$ $=0.4148$ ).

\section{Concluding Remarks}

In the past, the standard method intervals such as the score interval (SC) and the Wald interval with continuity correction interval (WCC) based on normal approximations, are both outperformed for moderate parameter mean and the sample size should be large enough $[2,3,5$, 7]. In this paper, the proposed alternative interval based on estimate tail probability, is called AWC interval. 
Table 1. Estimated $95 \%$ coverage probabilities and average widths for poisson means.

\begin{tabular}{|c|c|c|c|c|c|c|c|c|c|}
\hline \multirow{2}{*}{$\mathbf{n}$} & \multirow{2}{*}{ Mean $(\lambda)$} & \multicolumn{4}{|c|}{ Estimated Coverage Probability } & \multicolumn{4}{|c|}{ Estimated Average Width } \\
\hline & & SC & MSC & WCC & AWC & SC & MSC & WCC & AWC \\
\hline \multirow[t]{4}{*}{15} & 1 & 0.9494 & 0.9494 & 0.9801 & 0.9494 & 0.9804 & 0.9804 & 1.2142 & 0.9494 \\
\hline & 1.5 & 0.9423 & 0.9423 & 0.9718 & 0.9423 & 1.1844 & 1.1844 & 1.3914 & 1.1594 \\
\hline & 3 & 0.9570 & 0.9570 & 0.9646 & 0.9570 & 1.6898 & 1.6898 & 1.8277 & 1.6719 \\
\hline & 10 & 0.9551 & 0.9510 & 0.9530 & 0.9461 & 3.0635 & 3.0513 & 3.1269 & 3.0247 \\
\hline \multirow[t]{5}{*}{25} & 1 & 0.9437 & 0.9437 & 0.9847 & 0.9437 & 0.7489 & 0.7489 & 0.9451 & 0.7347 \\
\hline & 1.5 & 0.9508 & 0.9508 & 0.9734 & 0.9508 & 0.9209 & 0.9209 & 1.0797 & 0.9091 \\
\hline & 3 & 0.9433 & 0.9502 & 0.9640 & 0.9433 & 1.2864 & 1.2968 & 1.4146 & 1.2782 \\
\hline & 5 & 0.9477 & 0.9530 & 0.9612 & 0.9477 & 1.6660 & 1.6761 & 1.7677 & 1.6596 \\
\hline & 10 & 0.9476 & 0.9511 & 0.9534 & 0.9476 & 2.3517 & 2.3611 & 2.4221 & 2.3472 \\
\hline \multirow[t]{5}{*}{50} & 1 & 0.9454 & 0.9535 & 0.9853 & 0.9454 & 0.5275 & 0.5326 & 0.6689 & 0.5224 \\
\hline & 1.5 & 0.9450 & 0.9514 & 0.9738 & 0.9450 & 0.6444 & 0.6492 & 0.7637 & 0.6403 \\
\hline & 3 & 0.9554 & 0.9508 & 0.9655 & 0.9459 & 0.9192 & 0.9151 & 1.0012 & 0.9071 \\
\hline & 5 & 0.9473 & 0.9509 & 0.9600 & 0.9473 & 1.1757 & 1.1804 & 1.2482 & 1.1734 \\
\hline & 10 & 0.9490 & 0.9490 & 0.9560 & 0.9490 & 1.6647 & 1.6647 & 1.7173 & 1.6631 \\
\hline \multirow[t]{4}{*}{100} & 1 & 0.9510 & 0.9510 & 0.9861 & 0.9510 & 0.3741 & 0.3741 & 0.4735 & 0.3723 \\
\hline & 1.5 & 0.9571 & 0.9522 & 0.9764 & 0.9475 & 0.4603 & 0.4582 & 0.5412 & 0.4543 \\
\hline & 3 & 0.9471 & 0.9506 & 0.9648 & 0.9471 & 0.6438 & 0.6463 & 0.7077 & 0.6428 \\
\hline & 5 & 0.9489 & 0.9489 & 0.9602 & 0.9489 & 0.8324 & 0.8324 & 0.8828 & 0.8316 \\
\hline
\end{tabular}

From the simulation results (Table 1), the estimated 95\% coverage probabilities of AWC close to the nominal level in all cases and are similar to the SC and MSC interval. Morever, in many cases of small mean and small to moderate sample sizes such as $\lambda=1,1.5$ and $\mathrm{n}=15,25$, AWC seems to be preferable in the sence that their average width is shorter than others. However, when the mean and sample size are both increase, the average widths AWC interval are similar to other confidence intervals.

Therefore, it can be recommended that the AWC interval is more likely to be outperformed for small mean Poisson and small to moderate sample sizes. In addition, the AWC formula is simple and easy to compute. It should be considered that a sample observation is drawn from a Poisson distribution.

\section{REFERENCES}

[1] T. T. Cai, "One-Sided Confidence Intervals in Discrete Distributions," Journal of Statistical Planning and Inference, Vol. 131, No. 1, 2005, pp. 63-88. doi:10.1016/j.jspi.2004.01.005

[2] J. Byrneand and P. Kabaila, "Comparison of Poisson Con fidence Intervals," Communications in Statistics-Theory and Methods, Vol. 34, No. 3, 2005, pp. 545-556.

doi:10.1081/STA-200052109

[3] Y. Guan, "Moved Score Confidence Intervals for Means of Discrete Distributions," American Open Journal Statistics, Vol. 1, 2001, pp. 81-86. doi:10.4236/ojs.2011.12009

[4] K. Krishnamoorthy and J. Peng, "Improved Closed-Form Prediction Intervals for Binomial and Poisson Distribution," Journal of Statistical Planning and Inference, Vol. 141, No. 5, 2011, pp. 1709-1718. doi:10.1016/j.jspi.2010.11.021

[5] J. Stamey and C. Hamillton, "A Note on Confidence Intervals for a Linear Function of Poisson Rates," Communications in Statistics-Theory and Methods, Vol. 35, No. 4, 2005, pp. 849-856. doi:10.1080/03610920802255856

[6] M. B. Swifi, "Comparison of Confidence Intervals for a Poisson Mean-Further Considerations," Communications in Statistics-Theory and Methods, Vol. 38, No. 5, 2009, pp. 748-759.

[7] L. A. Barker, "Comparison of Nine Confidence Intervals for a Poisson Parameter When the Expected Number of Events Is $\leq 5$," The American Statistician, Vol. 56, No. 2, 2002, pp. 85-89. doi:10.1198/000313002317572736

[8] R Development Core Team, "R: A Language and Environment for Statistical Computing," R Foundation for 
Statistical Computing, Vienna, 2011.

[9] N. Gurtler and N. Henze, "Recent and Classical Goodness-of-Fit Tests for the Poisson Distribution," Journal of Statistical Planning and Inference, Vol. 90, No. 2, 2000, pp. 207-225. doi:10.1016/S0378-3758(00)00114-2

[10] P. Blaesild and J. Granfeldt, "Statistics with Applications in Biology and Geology," Chapman \& Hall/CRC, New York, 2003. 